\title{
Photothermal cantilever deflection spectroscopy
}

\author{
Seonghwan Kim', Dongkyu Lee ${ }^{2}$ and Thomas Thundat ${ }^{2^{*}}$
}

\author{
* Correspondence: \\ thundat@ualberta.ca \\ ${ }^{2}$ Department of Chemical and \\ Materials Engineering, University of \\ Alberta, T6G 2V4 Edmonton, AB, \\ Canada \\ Full list of author information is \\ available at the end of the article
}

\begin{abstract}
Microcantilever sensors offer high sensitivity in the detection of adsorbed molecules based either on resonance frequency shift or changes in cantilever deflection, as both of these signals can be detected with very high resolution. Despite the high sensitivity offered by this platform, cantilevers suffer from poor selectivity due to the lack of sufficiently selective interfacial layers which can be immobilized on cantilever surfaces. This problem can be overcome by using photothermal cantilever deflection spectroscopy (PCDS), which exploits the high thermomechanical sensitivity of bi-material microcantilevers. A bi-material cantilever responds to heat generated by the nonradiative decay process when the adsorbed molecules are resonantly excited with infrared (IR) light. The variation in the cantilever deflection as a function of illuminating IR wavelength corresponds to the conventional IR absorption spectrum of the adsorbed molecules. In addition, the mass of the adsorbed molecules can be determined by measuring the resonance frequency shift of the cantilever as an orthogonal signal for the quantitative analysis. This multi-modal PCDS offers unprecedented opportunities for obtaining very high selectivity in chemical and biological sensing without using selective interfacial layers or extrinsic labels.
\end{abstract}

Keywords: Bi-material microcantilever; Photothermal cantilever deflection spectroscopy; Nanomechanical IR spectrum; Explosives; Bitumen; Naphtha; DNA

\section{Introduction}

Recently, microcantilever sensors have attracted much attention due to their extremely high sensitivity [1,2]. These cantilevers, which can be microfabricated into arrays using conventional micromachining techniques, offer a miniature sensing platform for the real-time, simultaneous detection of multiple target analytes using a single device [3,4]. Another attractive feature of cantilever sensors is they can be operated in multiple modes. For example in the static mode, when confined to a single side of the cantilever, molecular adsorption results in cantilever deflection due to adsorption-induced forces [5]. In the dynamic mode, the resonance frequency of the cantilever varies sensitively as a function of adsorbed mass [5,6].

\section{Molecular adsorption-induced resonance frequency variation approach}

The resonance frequency, $f$, of a vibrating cantilever can be expressed as

$$
f=\frac{1}{2 \pi} \sqrt{\frac{k}{m^{*}}}
$$

where $k$ is the spring constant and $m^{*}$ is the effective mass of the microcantilever. The effective mass can be related to the mass of the beam, $m_{b}$, through the relation, $m^{*}=n$

\section{照 Springer}


$m_{b}$ where $n$ is a geometric parameter. For a rectangular cantilever, $n$ is 0.24 . It is also possible that chemisorption or chemical reaction of the adsorbed ions/molecules may alter the spring constant of the cantilever [7]. Therefore, from equation 1 it is clear the resonance frequency can vary due to changes in mass as well as changes in spring constant. The variation in the resonance frequency, therefore, can be generalized as:

$$
d f=\frac{f}{2}\left[\frac{d k}{k}-\frac{d m^{*}}{m^{*}}\right]
$$

The change in spring constant can be a result of changes in Young's modulus, E, or to changes in dimensions caused by the molecular absorption-induced swelling of the chemoselective interfacial layer such as a polymer film on the cantilever [8]. If the molecular adsorption can be confined to the terminal end of the cantilever (end loading), the contribution from the spring constant variation can be neglected. In that case, equation 1 is valid. Assuming the target molecules are evenly adsorbed on the cantilever surface and do not affect the stiffness of the cantilever, the adsorbed mass of the target molecules in air, $\delta m$, can be calculated from the simple equation:

$$
\delta m=\frac{k}{(2 \pi)^{2} n}\left(\frac{1}{f_{1}^{2}}-\frac{1}{f_{0}^{2}}\right)
$$

where, $f_{1}$ is the measured resonance frequency with adsorbed molecules, and $f_{0}$ is the initial resonance frequency of the cantilever [5].

\section{Molecular adsorption-induced cantilever deflection approach}

Thin microcantilevers undergo bending as a result of the mechanical forces involved in molecular adsorption. Adsorption-induced surface stress and the radius of the curvature of a cantilever can be related through Stoney's formula and the differential surface stress created by molecular adsorption can be related with the cantilever deflection as:

$$
\Delta z=\frac{3 L^{2}(1-v)}{E t^{2}} \delta \sigma
$$

where $L$ and $t$ are the length and the thickness of the cantilever, respectively, $v$ is the Poisson's ratio of the cantilever, and $\delta \sigma$ is the differential surface stress between the functionalized and the passivated surfaces. Therefore, the deflection of the cantilever is directly proportional to the adsorption-induced surface stress [5]. This surface stress is expressed in units of $\mathrm{N} / \mathrm{m}$ or $\mathrm{J} / \mathrm{m}^{2}$.

For either mass or surface stress based detection, the chemical selectivity has been obtained by immobilizing chemically selective interfaces on the cantilever surface. However, this method offers only limited selectivity since most chemical interfaces based on reversible chemical interaction are not very selective [9-13]. In spite of recent advances in microfabricated cantilever sensors with extremely high sensitivity, most sensing applications are hampered by poor selectivity. This challenge can be traced back to fundamental limitations imposed by the chemistry of the molecular interactions which forms the basis for signal generation in currently used chemical sensors. Simple chemical interactions such as hydrogen bonding are far too general for providing selectivity. In addition, immobilized chemoselective interfaces have a limited shelf-life as they degrade over time resulting even poorer selectivity [9]. 
We identify the lack of chemical selectivity in small molecule detection as the main challenge in accepting microcantilever sensors in practical applications. The limited selectivity and sensitivity of chemical sensors stems from the fundamental concept involved in the current sensing process which operates by the "molecule detects molecule" principle of selectivity. Adsorption of analyte molecules on the chemoselective interface immobilized on the cantilever surface results in variations in its mass and surface stress. Many current nano and micro sensors suffer from similar selectivity challenge as they are based on monitoring the changes in one of its physical properties, such as adsorbed mass, temperature, surface stress, resistance, capacitance, or refractive index, due to molecular adsorption. The transducer response is then amplified for readout and display. Despite all the advances in micro/nanotechnology, the fundamental mechanism of selectivity in sensing has not yet changed. In fact, current advances in chemical sensors are focused on developing transducers, which can detect extremely small changes in physical properties as a result of molecular adsorption. As long as the fundamental working principle for signal generation remains the same, the advantages of micro/nanotechnology will remain untouched in overcoming the challenges of sensor selectivity. Without selectivity, all other advantages (high sensitivity, fast response time, decrease in size and power consumption, and the potential for simultaneous detection of multiple analytes) are of no practical use.

Designing highly selective molecular recognition layers that can be immobilized on a sensor surface for small molecule detection is a challenging task due to the limited number of chemical interactions that can serve as the basis for synthesizing chemoselective layers while satisfying the highly desirable sensor attribute of room temperature reversibility. One way of achieving selectivity in detection is by using arrays of cantilevers where each element is modified with partially selective interfaces [10-12]. The requirement of room temperature reversibility requires the use of weak chemical interactions, for example hydrogen bonding, between the target molecules and the chemoselective interfaces. If the response from each cantilever is unique for a given chemical, it is possible to analyze the response using pattern recognition algorithms. The responses can include rise and decay times, as well as amplitudes. However, molecular recognition based on chemical interfaces relying on weak interactions is not specific enough to produce unique response pattern even in an array format. Increasing the number of sensor elements in the array for pattern recognition analysis does not improve the selectivity [13].

Imparting chemical selectivity to cantilever sensors is a challenge and will require utilizing unique properties of cantilever sensors in order to develop an entirely novel approach. One of the most under exploited properties of a cantilever sensor is its extremely high sensitivity to temperature variation when it is fabricated as a bi-material beam. This extreme high thermomechanical sensitivity of a bi-material cantilever can be exploited for measuring the extremely small thermal changes due to nonradiative decay of molecular vibrations of the adsorbed molecules [14-25]. The molecular vibrations of the adsorbed molecules can be resonantly excited by illuminating using infrared (IR) light. This change in heat energy due to molecular excitations on the cantilever sensor results in cantilever deflection. The amplitude of the bi-material cantilever deflection as a function of an illuminating wavelength shows the amount of heat energy generated by the adsorbed molecules at that wavelength and matches very well 
with the IR absorption peaks of the adsorbed molecules. This photothermal cantilever deflection spectroscopy (PCDS) is a unique technique that combines the extremely high thermomechanical sensitivity of a bi-material cantilever with the selectivity of mid-IR spectroscopy to achieve selectivity and sensitivity in molecular recognition of adsorbed molecules. By observing the energy location of multiple peaks in the cantilever deflection, it is possible to identify different molecules.

In this review article, we describe the working principle of PCDS, its instrumentation and methods. Quantitative results from the label-free, receptor-free detection of many analytes are presented. We also discuss potential strategies to enhance the thermomechanical sensitivity of bi-material cantilevers.

\section{Review}

The PCDS setup and its working principle are shown in Figure 1. Vapor phase analyte molecules are first allowed to adsorb on the bi-material cantilever surface. The mass of the adsorbed molecules is then calculated from the initial and final resonance frequencies of the cantilever. The cantilever is then illuminated with IR light from either a monochromator with a glow bar and a filter wheel or a broadly tunable quantum cascade laser (QCL). When IR photons of specific wavelength are resonantly absorbed by the adsorbates on the bi-material cantilever, it results in the generation of heat causing instantaneous cantilever deflection. The cantilever bends in response to this variation in temperature. By using a differential technique (cantilever with and without adsorbed molecules), it is possible to obtain a nanomechanical IR absorption spectrum of the adsorbates by plotting the differential cantilever deflection as a function of illumination wavelength. This signal depends on the thermodynamic and energy transfer properties of the adsorbates and the cantilever beam. Temperature changes resulting from the

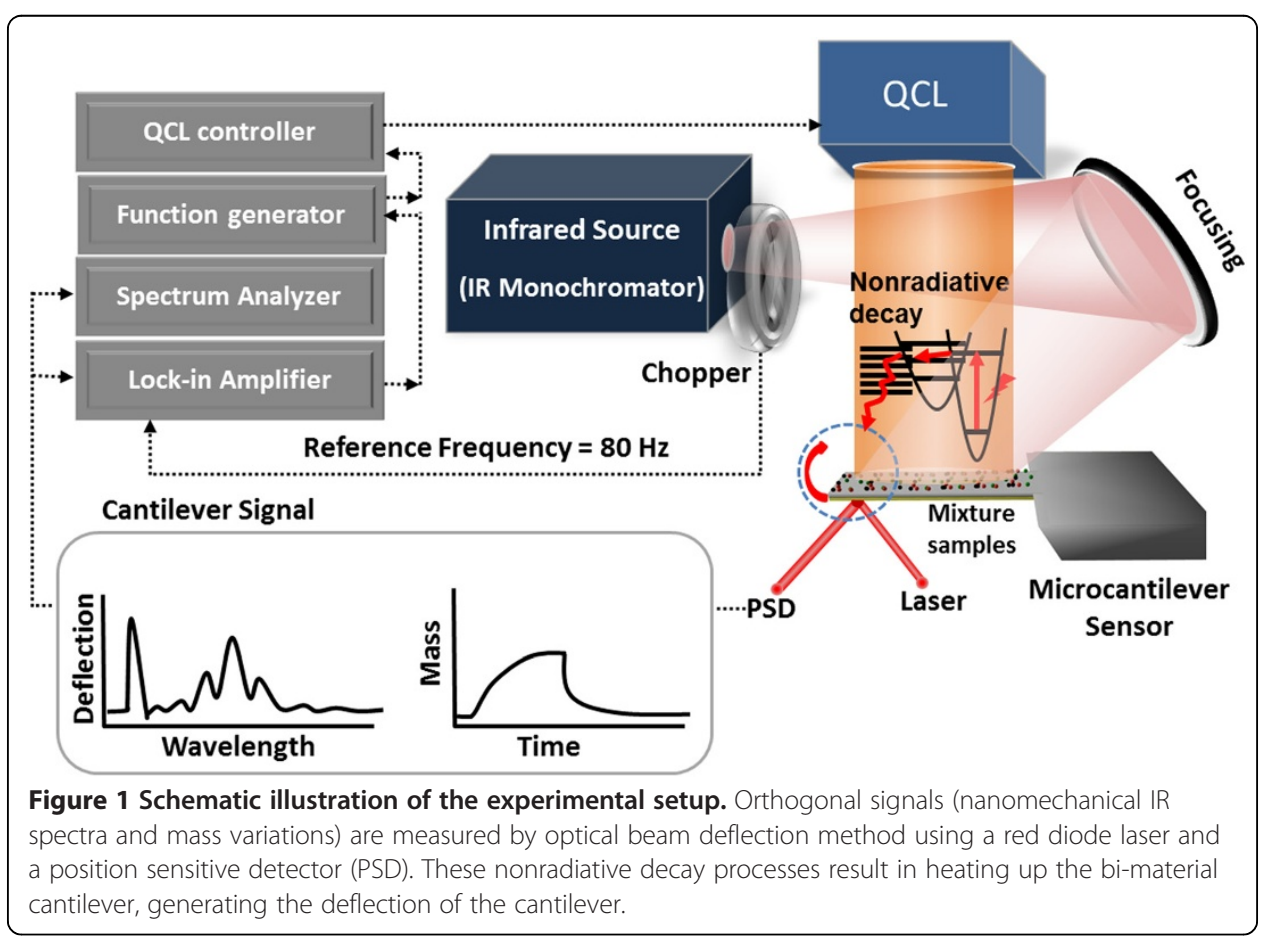


absorption of IR energy are directly related to the vibrational modes of the adsorbed molecules as well as to the heat capacity and thermal conductivity of the cantilever beam. The observed peak amplitudes of nanomechanical IR spectra are proportional to the amount of the adsorbed molecules, the impinging power of IR radiation, the absorption mode, and the thermomechanical sensitivity of the bi-material cantilever. Since the PCDS is based on nonradiative decay, it does not rely on Beer-Lambert's principle. Unlike, techniques based on Beer-Lambert's principle, such as Fourier transform infrared (FTIR) and conventional IR spectroscopy, the signal-to-noise ratio of PCDS increases with the power of the illumination source. Therefore, by increasing the power of the IR light source, it is possible to increase the sensitivity of the technique [21].

Since the resonance frequency of the cantilever can be monitored before and after molecular adsorption, the adsorbed mass can be simultaneously determined using equation 3. Therefore, within a certain dynamic range, the peak amplitudes of the nanomechanical IR spectra can be normalized by adsorbed mass and these can serve as basis for quantitative nanomechanical IR spectral analysis to determine the adsorbed mass of each target molecule in a mixture since the IR spectrum of a mixture is a linear superposition of individual spectra.

Since IR spectra are different for different molecules, high chemical selectivity can be achieved in detection. This technique can be used for selective detection and quantification of the binary and ternary mixtures of explosive molecules such as trinitrotoluene (TNT), cyclotrimethylene trinitramine (RDX), and pentaerythritol tetranitrate (PETN)) [21]. Figure 2 shows the normalized nanomechanical IR absorption spectra of explosive molecules. Figure 2a presents the normalized IR spectra of 1:1 binary mixtures such as TNT\&RDX (blue), PETN\&TNT (cyan), and RDX\&PETN (magenta). The nanomechanical IR spectra of the individual explosives were taken separately as references and agreed quite well with our previous report [20]. Figure $2 \mathrm{~b}$ shows the normalized nanomechanical IR absorption spectra of individual explosive molecules, TNT (black), PETN (red), and RDX (green), using a conventional monochromator. The signal amplitude is in the range of $\mathrm{mV} / \mathrm{ng}$. However, the amplitude of normalized nanomechanical IR absorption spectra for the same samples using the QCL in Figure 2c were three orders of magnitude higher than that of spectra using a monochromator in Figure 2b. The sensitivity of PCDS was improved by employing a broadly tunable QCL as a powerful IR source [21]. Several distinct peaks and shoulders appeared in the binary mixture spectrum since the mixture spectrum is a linear superposition of individual spectra. Comparing these peaks with those of individual TNT, RDX, and PETN spectra, it is apparent that the peaks at 6.49 and $7.46 \mu \mathrm{m}$ are from TNT, the peaks at 6.38 and $7.27 \mu \mathrm{m}$ are from RDX, and the peaks at 6.06 and $7.82 \mu \mathrm{m}$ are from PETN molecules [26-30]. PCDS can detect and distinguish differences between such closely related molecular species and between other interfering compounds and target molecules while sensing in mixture samples.

Selective and sensitive detection has direct relevance in many industrial applications such as chemical process and environmental monitoring [22,31]. For example, naphtha - a complex hydrocarbon - is routinely used for extraction of bitumen from oil sands, needs to be monitored on a routine basis in oil sands processing. Since the process of stripping naphtha from bitumen is not perfect, they easily lost into tailing streams during bitumen extraction process. However, the detection of industrial solvent and 


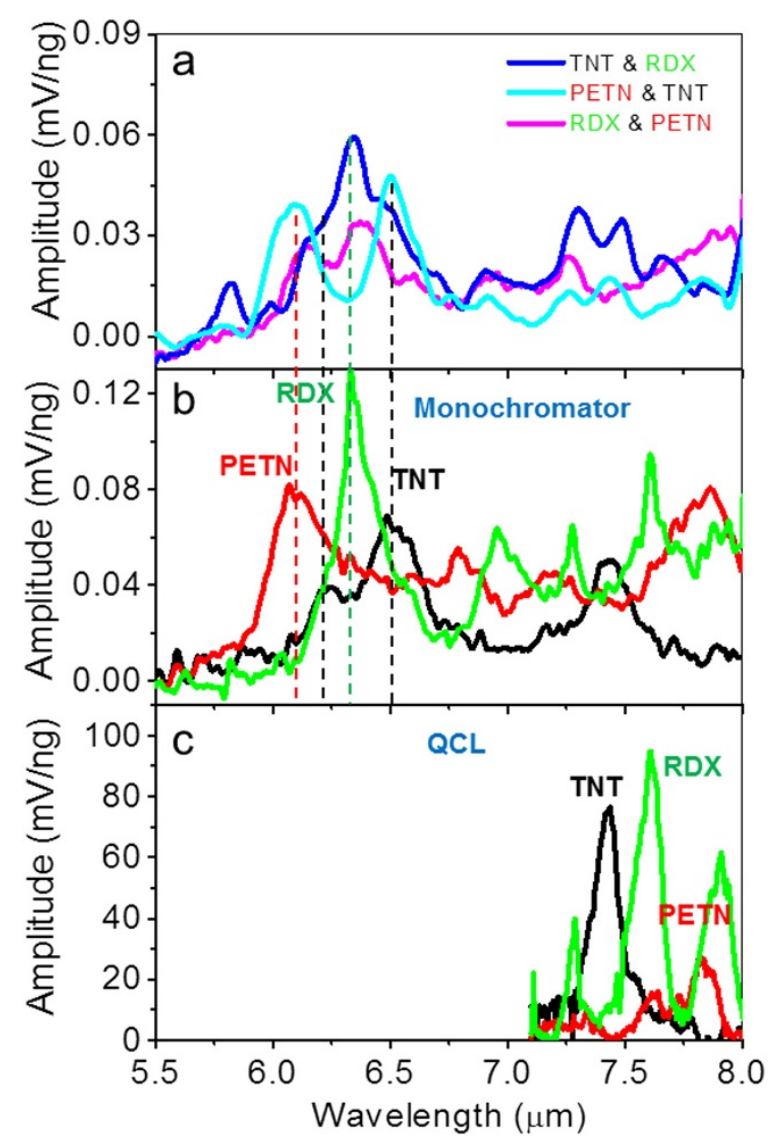

Figure 2 Normalized nanomechanical IR absorption spectra of explosive molecules. (a) Normalized IR spectra of 1:1 binary mixtures (TNT\&RDX (blue), PETN\&TNT (cyan), RDX\&PETN (magenta)), and three individual explosive component (TNT (black), PETN (red), RDX (green)) using (b) monochromator-based and (c) QCL-based PCDS.

chemicals such as high volatile organic compounds poses many challenges as a result of non-specific interactions with surfaces and interferences from identical groups in other compounds. By monitoring multiple peaks of adsorbed molecules in PCDS, it is possible to overcome the selectivity and sensitivity challenges of currently used techniques. Figure 3 demonstrates selective detection of naphtha and bitumen using PCDS. The IR spectra of bitumen and naphtha reveal that they are largely composed of the aliphatic compounds. The common bands correspond to $\mathrm{C}-\mathrm{H}$ asymmetrical stretching of $-\mathrm{CH}_{2}$ and $-\mathrm{CH}_{3}$ in range of 3.35 to $3.5 \mu \mathrm{m}$. The two bands at 6.8 and $7.2 \mu \mathrm{m}$ are associated with $\mathrm{C}-\mathrm{H}$ deformation in $-\mathrm{CH}_{2}$ and $-\mathrm{CH}_{3}$, respectively. Carbon rings in a cyclic compound and $\mathrm{CH}$ out of plane deformations in vinyl compounds show peaks in the range of 9.7-10.3 $\mu \mathrm{m}[22,31]$. However, we can clearly distinguish them with some characteristic peaks, which can be attributed to different ratio of each component and some minor mixture components having various functional groups such as carboxylic acids $(\mathrm{C}-\mathrm{O}, \mathrm{C}-\mathrm{O}-\mathrm{H})$ and ring vibrations $(\mathrm{C}=\mathrm{C}, \mathrm{C}-\mathrm{C})$.

The PCDS technique also offers high selectivity in detection of biological samples. Despite intrinsic selectivity in the binding of antigen-antibody or DNA hybridization, the microfabricated biosensors incur several problems such as non-uniform 


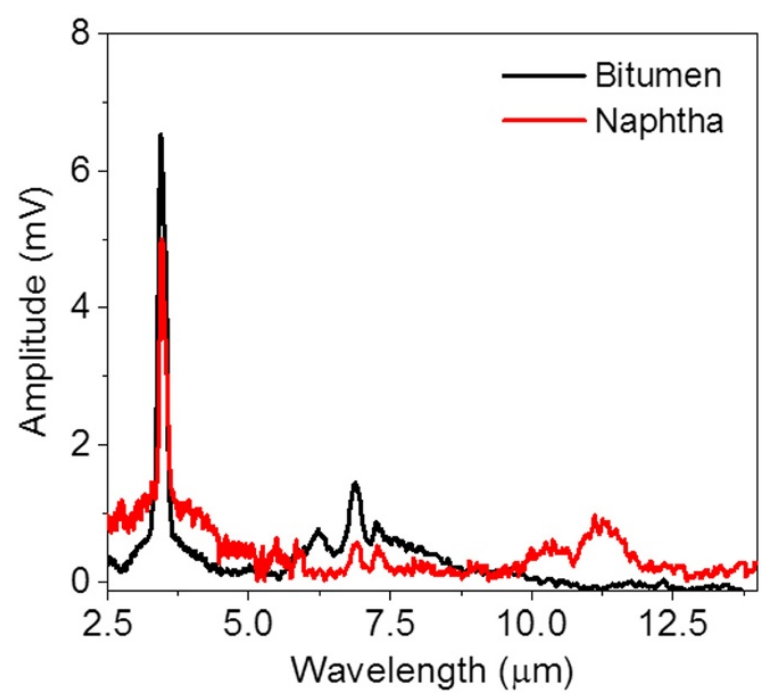

Figure 3 Nanomechanical IR absorption spectra of bitumen (black) and naphtha (red).

immobilization, reduced activity of receptors, and non-specific binding of unwanted molecules. Additionally, it is not easy to rapidly detect biological target molecules due to the intensive sample delivery process and reaction time involved in the receptortarget interactions. To overcome these issues, the PCDS technique can be used as an effective label-free and receptor-free biosensing concept. Figure 4 shows the normalized nanomechanical IR absorption spectra of the ssDNA of 20 bases of guanine using a conventional monochromator (Figure 4a). Figure $4 \mathrm{~b}$ shows the same ssDNA spectrum obtained using a QCL which can scan from $5.68 \mu \mathrm{m}$ to $6.39 \mu \mathrm{m}$. In Figure 4, the peaks between 5.68 and $6.39 \mu \mathrm{m}$ result from the stretching vibration of the carbonyl group and ring bonds in guanine. The peaks of guanine are from carbonyl $\mathrm{C}=\mathrm{O}$ displacement $(5.91 \mu \mathrm{m})$ and ring bond $\mathrm{C}=\mathrm{N}$ stretching $(6.1,6.25 \mu \mathrm{m})$ [32,33]. The sensitivity of QCL-based PCDS was found to be superior to that of monochromator-based PCDS as a result of using a high power IR source. These experiments show that QCL-based PCDS has the great potential for rapid identification and quantification of biological analytes. Although this technique is not able to discern different DNA sequences, it is highly useful as a rapid screening method for potential variation (mutation) in a given DNA strand with a known-sequence as well as detection of conjugation and byproduct of biomolecular interactions $[32,34,35]$. This technique may also find forensic applications where the need is to discriminate DNA rather than sequencing.

The relative intensities of the PCDS peaks were different than those observed with conventional IR spectrum or FTIR method. Some peaks that are not very prominent in conventional IR spectra appear to have higher intensity in the PCDS spectra. This may be directly related to the efficiency of nonradiative decay of these excited states. The position of absorption peaks were in general agreement with those observed with conventional IR absorption spectroscopies. We did not observe any appreciable shift in the position of the PCDS peaks. Since the energy resolution of the PCDS depends on the energy resolution of the IR sources, any small shift induced by the presence of the substrate is below the resolution limit of the current device. 


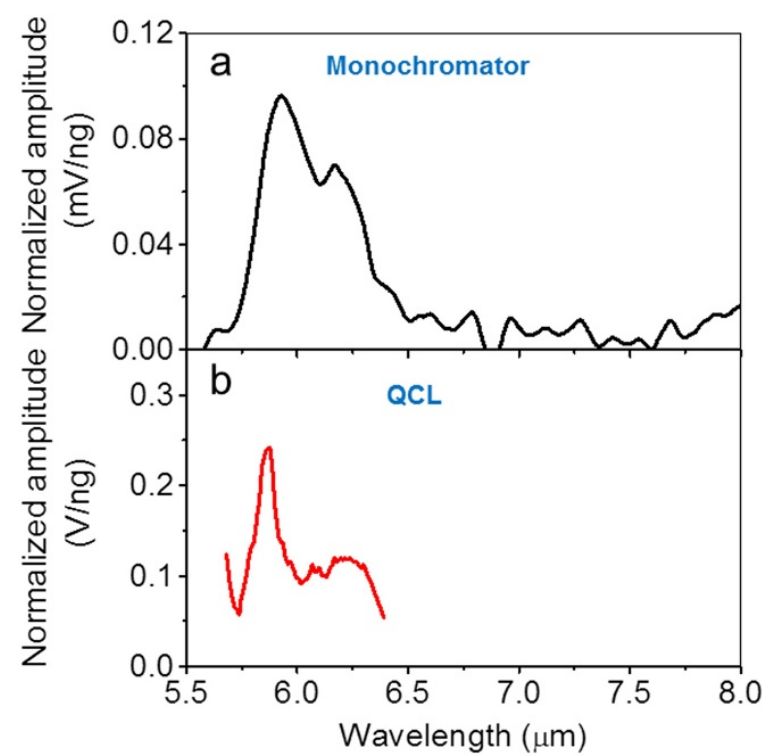

Figure 4 Normalized nanomechanical IR absorption spectra of ssDNA of 20-mer guanine using monochromator-based (a) and (b) QCL-based PCDS.

\section{Advantages of the PCDS technique}

The PCDS does not use any chemical receptors or interfaces for molecular recognition. The cantilever used in this technique has no surface functional groups. The molecules can be deposited on the surface with no chemical interaction between the analyte and the substrate. Even placing a drop of solution and drying it under ambient conditions is sufficient to obtain PCDS signals.

- High Selectivity: The signal generation is based on resonance excitation of various molecular bonds in the analyte. Molecular vibrations are extremely selective. In addition, in a complex mixture, the resultant signal is a linear combination of molecular vibrations from various constituents. Therefore, using a simple superposition principle it is possible to analyze the signal for the target analyte.

- High Sensitivity: The PCDS combines the extreme high thermomechanical sensitivity of a bi-material cantilever beam with the selectivity of mid-IR molecular spectroscopy. Our earlier results have shown mass sensitivity of $10 \mathrm{pg}$ on the cantilever (corresponding to a few monolayers of analyte) under ambient conditions.

- Quantitative: The magnitude of the IR absorption-induced cantilever bending is proportional to the amount of analyte on the cantilever. This can be further quantified by measuring the resonance frequency of the cantilever (mass loading).

- Real-time Monitoring: Since the heat generation due to non-radiative decay is instantaneous, the PCDS technique is capable of real-time monitoring of adsorbed analytes.

- Small Size: The first generation device can be packaged to be smaller than a shoe box (including IR source, power supplies, display unit, etc.) making it a portable device. A second generation device can be made even smaller (the size of a cell phone) by optimizing packaging. 
- Low Cost: Once produced in bulk, the cost of the device will be a few thousand dollars.

- Versatility: The same device can be used for different analytes.

\section{Conclusions}

PCDS provides simple technique to impart chemical selectivity onto microcantilever sensors. The demonstrated sensitivity and selectivity of this approach offers new possibilities for receptor-free sensing of a wide range of materials beyond what is currently possible using conventional techniques. These devices have the obvious advantages of requiring sub-nanogram samples, quick detection time and the potential to be inexpensive. It is possible to increase the sensitivity of PCDS by optimizing the bi-material cantilever parameters as well as increasing the power of the illuminating IR source. For example, by selecting different metals and optimizing the thickness of the coating it is possible to make a bi-material cantilever very sensitive to thermal changes. It is also possible to fabricate cantilevers with an optimized spring constant for increased thermomechanical bending or to pattern the cantilever surface for increased adsorption. Additionally, sensitivity can be increased by restricting the heat flow from the cantilever onto the base of the cantilever by making the contact area smaller.

\section{Method}

\section{Maximizing the thermomechanical sensitivity of a bi-material cantilever}

A microcantilever which is made bi-material by depositing a thin layer of metal on one of its side becomes an excellent, miniature thermal sensor, capable of detecting extremely small temperature changes at room temperature (there is no need to cool the device). The deflection of the cantilever tip due to the bi-material effect can be found from the following equation [36]:

$$
z=-\frac{3}{4}\left(\alpha_{1}-\alpha_{2}\right) \frac{t_{1}+t_{2}}{t_{2}^{2} K} \frac{l^{3}}{\left(\lambda_{1} t_{1}+\lambda_{2} t_{2}\right) w} P
$$

where $z$ is the cantilever deflection at the tip, $\alpha_{1}$ and $\alpha_{2}$ are the coefficients of thermal expansion for the two layers, $l$ is the length of the cantilever, $t_{1}$ and $t_{2}$ are the layer thicknesses, $\lambda_{1}$ and $\lambda_{2}$ are the thermal conductivities, $w$ is the width of the cantilever, and $P$ is the total power absorbed by the cantilever. The subscripts 1 and 2 represent metal and cantilever substrate, respectively. The parameter $K$ stands for the expression:

$$
K=4+6\left(\frac{t_{1}}{t_{2}}\right)+4\left(\frac{t_{1}}{t_{2}}\right)^{2}+\frac{E_{1}}{E_{2}}\left(\frac{t_{1}}{t_{2}}\right)^{3}+\frac{E_{2}}{E_{1}}\left(\frac{t_{2}}{t_{1}}\right) .
$$

In the PCDS technique, the cantilever with adsorbed chemical species is scanned using successive pulses of monochromatic IR radiation. The amplitude of cantilever deflection as a function of IR wavelength resembles the IR absorption spectrum of the adsorbed molecules. The limit of detection of this technique is determined by the power of IR source and the thermomechanical sensitivity of the cantilever, which can be optimized by properly designing the bi-material aspect of the cantilever. 


\section{Instrumentation}

\section{Preparation of bi-material cantilevers}

The experiments were carried out using commercially available silicon microcantilevers. Rectangular silicon cantilevers (CSC12-E) were obtained from MikroMasch USA (San Jose, CA). The dimension of each cantilever was $350 \mu \mathrm{m}$ in length, $35 \mu \mathrm{m}$ in width, and $1 \mu \mathrm{m}$ in thickness. Another rectangular silicon cantilevers (Octosensis) were obtained from Micromotive GmbH Germany. A silicon chip contained an array of eight cantilevers having typical dimensions of $500 \mu \mathrm{m}$ in length, $90 \mu \mathrm{m}$ in width, and $1.0 \pm$ $0.3 \mu \mathrm{m}$ in thickness. The microcantilevers were cleaned by rinsing with acetone, ethanol, and treating them with UV ozone and then coated with $10 \mathrm{~nm}$ of chromium (adhesion layer) followed by $200 \mathrm{~nm}$ of gold using an e-beam evaporator at the deposition rate of $0.2 \AA / \mathrm{s}$. A bare Octosensis silicon cantilever was used for bitumen experiments. Bitumen was diluted with toluene and coated on top of the silicon cantilever using a micro glass capillary. The bitumen-coated cantilever was dried in a vacuum oven at $150^{\circ} \mathrm{C}$ overnight to remove any low molecular weight solvent mixed in with the bitumen. Three standard explosive samples (TNT, RDX, and PETN) were purchased from AccuStandard, Inc. (New Haven, CT) and used without further purification. As indicated by the manufacturer, the standard concentration of each explosive is $1 \mathrm{mg} / \mathrm{mL}$. The binary mixtures (by volume of standard sample solution) of explosive molecules were deposited on CSC12-E microcantilevers using micro glass capillaries. Naphtha was deposited on the gold side of the Octosensis cantilever by drop casting. The used chemicals (acetone, ethanol, toluene, and naphtha) were purchased from Fisher Scientific and used without further purification. We also procured synthetic single-stranded DNA (ssDNA) of 20 bases of guanine (G20) from Oligos Etc. The initial concentration of the ssDNA solution was $10 \mu \mathrm{M}$ in deionized water. DNAs were deposited on the cantilever by inserting the cantilever into a micro glass capillary containing the DNA solution and slowly taking the cantilever out of the capillary.

\section{PCDS setup}

The sample-coated cantilever was mounted on a stainless steel cantilever holder and then positioned in the head unit of a Multi-Mode atomic force microscope (AFM) (Bruker, Santa Barbara, CA). The nanomechanical deflection and resonance frequency of the cantilever were measured using the optical beam deflection method with a red laser diode and a position sensitive detector (PSD). The IR radiation from the monochromator (Foxboro Miran 1A-CVF) was mechanically chopped at a frequency of $80 \mathrm{~Hz}$ and focused onto the cantilever by a spherical mirror. The filter wheel of the monochromator scans from $2.5 \mu \mathrm{m}$ to $14.5 \mu \mathrm{m}$ (i.e., $4000 \mathrm{~cm}^{-1}$ to $690 \mathrm{~cm}^{-1}$ in wavenumber) and has a spectral resolution of approximately $0.05 \mu \mathrm{m}$ at $3 \mu \mathrm{m}, 0.12 \mu \mathrm{m}$ at $6 \mu \mathrm{m}$, and $0.25 \mu \mathrm{m}$ at $11 \mu \mathrm{m}$ according to the manufacturer. The $200 \mathrm{kHz}$ pulsed IR radiation with a $10 \%$ duty cycle from a ÜT-8 QCL (Daylight Solutions, San Diego, CA) was electrically modulated at $80 \mathrm{~Hz}$, using a DS345 function generator (Stanford Research Systems, Sunnyvale, CA) and directed to the cantilever for explosive sensing experiments. The IR wavelength was scanned from $7.1 \mu \mathrm{m}$ to $8.73 \mu \mathrm{m}\left(1408 \mathrm{~cm}^{-1}\right.$ to $1145 \mathrm{~cm}^{-1}$ ) with a step size of $5 \mathrm{~nm}$. For DNA sensing experiments, $100 \mathrm{kHz}$ pulsed IR radiation with a 5\% duty cycle from a QCL (Daylight Solutions $6 \mu \mathrm{m}$ laser) was electrically modulated at $80 \mathrm{~Hz}$ and directed to the DNA-coated cantilever. The IR wavelength 
was scanned from $5.68 \mu \mathrm{m}$ to $6.39 \mu \mathrm{m}\left(1760 \mathrm{~cm}^{-1}\right.$ to $\left.1565 \mathrm{~cm}^{-1}\right)$ with a step size of $5 \mathrm{~nm}$. The resonance frequencies of the cantilever were measured before and after sample deposition using an SR760 spectrum analyzer (Stanford Research Systems, Sunnyvale, CA). The nanomechanical IR deflection signals before and after sample deposition were taken using an SR850 lock-in amplifier (Stanford Research Systems, Sunnyvale, CA) and the differential signals were presented as the nanomechanical IR spectra.

\section{Abbreviations}

IR: Infrared; PCDS: Photothermal cantilever deflection spectroscopy; QCL: Quantum cascade laser; FTIR: Fourier transform infrared; TNT: Trinitrotoluene; RDX: Cyclotrimethylene trinitramine; PETN: Pentaerythritol tetranitrate.

\section{Competing interests}

The authors declare that they have no competing interests.

\section{Authors' contributions}

SK \& DL designed, carried out the experiments, collected and analyzed the data, and wrote the manuscript; $\Pi T$ conceived the experiments, analyzed results, and wrote the manuscript. All authors discussed the results and commented on the manuscript. All authors read and approved the final manuscript.

\section{Authors' information}

Seonghwan Kim is an Assistant Professor in the Department of Mechanical and Manufacturing Engineering at the University of Calgary. He earned his B.S. and M.S. degree in aerospace engineering at Seoul National University in 1998 and 2000, respectively, and received his Ph.D. in mechanical engineering at the University of Tennessee, Knoxville in 2008.

Dongkyu Lee is a Postdoctoral fellow in the Department of Chemical and Materials Engineering, University of Alberta, Edmonton. He received his Ph.D. in chemical engineering from Pohang University of Science and Technology, Pohang, Korea in 2011.

Thomas Thundat is a Canada Excellence Research Chair Professor at the University of Alberta, Edmonton. He received his Ph.D. in physics from the State University of New York at Albany in 1987.

\section{Acknowledgements}

This work is supported by Canada Excellence Research Chairs (CERC) Program. D.L. also would like to acknowledge the partial support from Basic Science Research Program through the National Research Foundation of Korea (NRF) funded by the Ministry of Education, Science and Technology (2012R1A6A3A03040416). SK also acknowledges the partial support from the Schulich School of Engineering in the University of Calgary.

\section{Author details}

${ }^{1}$ Department of Mechanical and Manufacturing Engineering, University of Calgary, T2N 1N4 Calgary, AB, Canada.

${ }^{2}$ Department of Chemical and Materials Engineering, University of Alberta, T6G 2V4 Edmonton, AB, Canada.

Received: 20 February 2014 Accepted: 24 June 2014

Published: 5 August 2014

\section{References}

1. Thundat T, Warmack RJ, Chen GY, Allison DP: Thermal and ambient-induced deflections of scanning force microscope cantilevers. Appl Phys Lett 1994, 64:2894-2896.

2. Gimzewski JK, Gerber C, Meyer E, Schlittler RR: Observation of a chemical reaction using a micromechanical sensor. Chem Phys Lett 1994, 217:589-594.

3. Baller MK, Lang HP, Fritz J, Gerber C, Gimzewski JK, Drechsler U, Rothuizen H, Despont M, Vettiger P, Battiston FM, Ramseyer JP, Fornaro P, Meyer E, Güntherodt HJ: A cantilever array-based artificial nose. Ultramicroscopy 2000, $82: 1-9$.

4. Fritz J, Baller MK, Lang HP, Rothuizen H, Vettiger P, Meyer E, Güntherodt HJ, Gerber C, Gimzewski JK: Translating biomolecular recognition into nanomechanics. Science 2000, 288:316-318.

5. Chen GY, Thundat T, Wachter EA, Warmack RJ: Adsorption-induced surface stress and its effects on resonance frequency of microcantilevers. J Appl Phys 1995, 77:3618-3622.

6. Thundat T, Chen GY, Warmack RJ, Allison DP, Wachter EA: Vapor detection using resonating microcantilevers. Anal Chem 1995, 67:519-521.

7. Cherian $\mathrm{S}$, Thundat T: Determination of adsorption-induced variation in the spring constant of a microcantilever. Appl Phys Lett 2002, 80:2219-2221.

8. Wachter EA, Thundat T: Micromechanical sensors for chemical and physical measurements. Rev Sci Instrum 1995, 66:3662-3667.

9. Hsieh MD, Zellers ET: Limits of recognition for simple vapor mixtures determined with a microsensor array. Anal Chem 2004, 76:1885-1895.

10. Then $D$, Vidic A, Ziegler $C$ : A highly sensitive self-oscillating cantilever array for the quantitative and qualitative analysis of organic vapor mixtures. Sens Actuators B 2006, 117:1-9.

11. Zhao W, Pinnaduwage LA, Leis JW, Gehl AC, Allman SL, Shepp A, Mahmud KK: Identification and quantification of components in ternary vapor mixtures using a microelectromechanical-system-based electronic nose. J Appl Phys 2008, 103:104902. 
12. Jin C, Kurzawski P, Hierlemann A, Zellers ET: Evaluation of multitransducer arrays for the determination of organic vapor mixtures. Anal Chem 2008, 80:227-236.

13. Jin C, Zellers ET: Limits of recognition for binary and ternary vapor mixtures determined with multitransducer arrays. Anal Chem 2008, 80:7283-7293.

14. Barnes JR, Stephenson RJ, Welland ME, Gerber C, Gimzewski JK: Photothermal spectroscopy with femtojoule sensitivity using a micromechanical device. Nature 1994, 372:79-81.

15. Wachter EA, Thundat T, Oden PI, Warmack RJ, Datskos PG, Sharp SL: Remote optical detection using microcantilevers. Rev Sci Instrum 1996, 67:3434-3439.

16. Li G, Burggraf LW, Baker WP: Photothermal spectroscopy using multilayer cantilever for chemical detection. Appl Phys Lett 2000, 76:1122-1124.

17. Datskos PG, Rajic S, Sepaniak MJ, Lavrik N, Tipple CA, Senesac LR, Datskou I: Chemical detection based on adsorption-induced and photoinduced stresses in microelectromechanical systems devices. J Vac Sci Technol B 2001, 19:1173-1179.

18. Arakawa ET, Lavrik NV, Rajic S, Datskos PG: Detection and differentiation of biological species using microcalorimetric spectroscopy. Ultramicroscopy 2003, 97:459-465.

19. Wig A, Arakawa ET, Passian A, Ferrell TL, Thundat T: Photothermal spectroscopy of Bacillus anthracis and Bacillus cereus with microcantilevers. Sens Actuators B 2006, 114:206-211.

20. Krause $A R$, Van Neste $C$, Senesac $L$, Thundat $T$, Finot E: Trace explosive detection using photothermal deflection spectroscopy. J Appl Phys 2008, 103:094906.

21. Kim S, Lee D, Liu X, Van Neste C, Jeon S, Thundat T: Molecular recognition using receptor-free nanomechanical infrared spectroscopy based on a quantum cascade laser. Sci Rep 2013, 3:1111.

22. Bagheri M, Chae I, Lee D, Kim S, Thundat T: Selective detection of physisorbed hydrocarbons using photothermal cantilever deflection spectroscopy. Sens Actuators B 2014, 191:765-769.

23. Lee D, Kim S, Van Neste CW, Lee M, Jeon S, Thundat T: Photoacoustic spectroscopy of surface adsorbed molecules using a nanostructured coupled resonator array. Nanotechnology 2014, 25:035501.

24. Khan MF, Kim S, Lee D, Schmid S, Boisen A, Thundat T: Nanomechanical identification of liquid reagents in a microfluidic channel. Lab Chip 2014, 14:1302-1307.

25. Lee D, Kim S, Chae I, Jeon S, Thundat T: Nanowell-patterned TiO2 microcantilevers for calorimetric chemical sensing. Appl Phys Lett 2014, 104:141903.

26. Pristera F, Halik M, Castelli A, Fredericks W: Analysis of Explosives Using Infrared Spectroscopy. Anal Chem 1960, 32:495-508.

27. Lewis IR, Daniel NW Jr, Griffiths PR: Interpretation of raman spectra of nitro-containing explosive materials. part i: group frequency and structural class membership. App/ Spectrosc 1997, 51:1854-1867.

28. Makashir PS, Kurian EM: Spectroscopic and thermal studies on 2,4,6-trinitro toluene (TNT). J Therm Anal Cal 1999, 55:173-185.

29. Makashir PS, Kurian EM: Spectroscopic and thermal studies on Pentaerythritol Tetranitrate (PETN). Propell Explos Pyrot 1999, 24:260-265.

30. Beal RW, Brill TB: Vibrational Behavior of the -NO2 Group in Energetic Compounds. Appl Spectrosc 2005, 59:1194-1202.

31. Yoon S, Son J, Lee W, Lee H, Lee CW: Prediction of bitumen content in oil sand based on FT-IR measurement. $J$ Ind Eng Chem 2009, 15:370-374.

32. Dovbeshko Gl, Gridina NY, Kruglova EB, Pashchuk OP: FTIR spectroscopy studies of nucleic acid damage. Talanto 2000, 53:233-246.

33. Kim S, Lee D, Thundat R, Bagheri M, Jeon S, Thundat T: Photothermal cantilever deflection spectroscopy. ECS Trans 2013, 50:459-464.

34. Mateo-Marti E, Briones C, Roman E, Briand E, Pradier CM, Martin-Gago JA: Self-assembled monolayers of peptide nucleic acids on gold surfaces: a spectroscopic study. Langmuir 2005, 21:9510-9517.

35. Liao W, Wei F, Liu D, Qian MX, Yuana G, Zhaoa XS: FTIR-ATR detection of proteins and small molecules through DNA conjugation. Sens Actuators B 2006, 114:445-450.

36. Lai J, Perazzo T, Shi Z, Majumdar A: Optimization and performance of high-resolution micro-optomechanical thermal sensors. Sens Actuators A 1997, 58:113-119.

\section{Submit your manuscript to a SpringerOpen ${ }^{\circ}$ journal and benefit from:}

- Convenient online submission

Rigorous peer review

- Immediate publication on acceptance

- Open access: articles freely available online

- High visibility within the field

- Retaining the copyright to your article

Submit your next manuscript at $\gg$ springeropen.com 